\title{
Measurement of the top-Higgs Yukawa coupling in the tHq process with CMS
}

\section{Pallabi Das*}

on behalf of the CMS Collaboration

Tata Institute of Fundamental Research, Mumbai-400005, India

E-mail: pallabi.das@cern.ch

\begin{abstract}
The current mandate of the LHC experiments is to precisely determine the properties of the Higgs boson, one of the major interests being the top-Yukawa coupling $\left(y_{t}\right)$ measurement. The Run 2 data have provided evidence of $\bar{t}_{\mathrm{t}} \mathrm{H}$ production at the LHC which is sensitive to $y_{t}^{2}$ at the tree level. In contrast, the tHq process provides information on the relative sign of $y_{t}$ with respect to the Higgs to vector boson coupling $\left(g_{H V V}\right)$. According to the Standard Model (SM), the cross section of the tHq process is very low even at $13 \mathrm{TeV}$ centre of mass energy $(\sqrt{s})$. However, in case of anomalous Higgs boson couplings the cross section may be enhanced and be detected with a limited amount of integrated luminosity $(L)$. Results are presented from Run 2 measurements of the tHq process using LHC data collected by the CMS experiment. Future prospects of the search are discussed in the context of High Luminosity LHC (HL-LHC).
\end{abstract}

European Physical Society Conference on High Energy Physics - EPS-HEP2019 -

10-17 July, 2019

Ghent, Belgium

\footnotetext{
*Speaker.
} 


\section{Introduction}

After the discovery of the Higgs boson in 2012 [1, 2, 3] by the ATLAS [4] and CMS [5] collaborations, the precise measurements of its properties have gained paramount importance. The SM has accurate predictions for the Higgs boson couplings, given its mass which has been measured with better than $2 \%$ precision [6] using Run 1 LHC data. In this context, the interpretations are provided in terms of coupling modifiers with respect to SM values: $\kappa_{V}$ and $\kappa_{t}$ for $g_{H V V}$ and Yukawa couplings $y_{f}$ respectively. One of the major highlights of Run 2 is the observation of the t⿱亠t $\mathrm{H}$ process $[18,19]$ which probes the top-Yukawa coupling at the tree level. However, the $\bar{t} \overline{\mathrm{t}} \mathrm{H}$ production is only sensitive to the magnitude of the coupling as its cross section varies as $y_{t}^{2}$. A complementary measurement is possible using the sub-dominant single top associated production of the Higgs boson (tH) which is sensitive to the relative sign of $\kappa_{t}$ with respect to $\kappa_{V}$ due to interfering leading order diagrams as shown in Figs. 1 and 2.
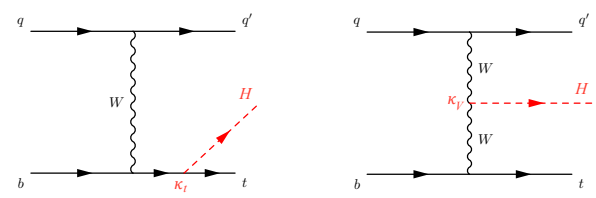

Figure 1: Feynman diagrams contributing to the t-channel tH production.

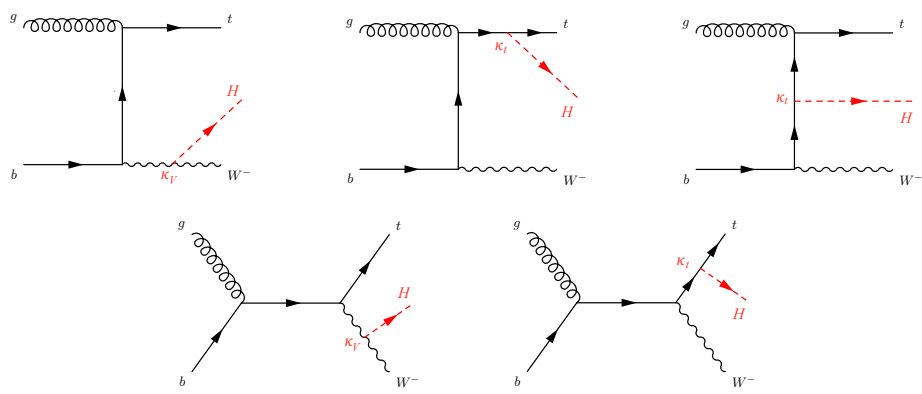

Figure 2: Feynman diagrams contributing to the $\mathrm{W}$-associated tH production.

The dominant $\mathrm{t}$-channel production mode tHq has a cross section of $\sim 71 \mathrm{fb}$ for pp collisions at $\sqrt{\mathrm{s}}=13 \mathrm{TeV}$. The rate of $\mathrm{W}$-associated production tHW is even smaller, $\sim 15 \mathrm{fb}$. Due to effects beyond the SM, anomalous couplings of the Higgs boson can result in large enhancements in the cross sections of both tH production channels. For example, in the inverted top coupling (ITC) scenario i.e., $\kappa_{t}=-\kappa_{V}=-1$, the cross section is enhanced by more than ten folds. From LHC Run 1 data a $95 \%$ confidence level (CL) upper limit on tHq production in the ITC scenario was extracted for the first time [7] using $L \sim 20 \mathrm{fb}^{-1}$ at $\sqrt{s}=8 \mathrm{TeV}$ yielding an observed upper limit twice that of the expectation. With the increasing amount of data volume available in Run 2, additional coupling scenarios for the Higgs boson has been probed following the Higgs characterization model [8, 9]. The analyses presented here are based on $L \sim 36 \mathrm{fb}^{-1}$ of data collected by the CMS experiment in 2016 at $\sqrt{s}=13 \mathrm{TeV}$. 


\section{Multilepton channel}

The tHq multilepton final state analysis [11] considers Higgs decays to $\mathrm{WW}^{*}$ and semileptonic decay of the top, resulting in two event categories based on lepton multiplicity: exactly two same sign leptons ( $2 \ell s s)$ or three leptons $(3 \ell)$ in addition to at least one b-tagged jet and at least one non b-tagged jet.

There are two major types of background to the leptonic final state, one originating from prompt leptons (mainly ttitW and $\bar{t} \bar{Z} Z$ events) and the second consisting of non-prompt leptons or fakes (mainly from $t \bar{t}+j e t s$ ) which may be mis-identified jets or real leptons from $b / c$ hadron decays. Selection criteria for well-reconstructed and isolated leptons to efficiently reject non-prompt leptons and estimate their contribution to background follow the t⿱亠t十H multilepton analysis [10].

A multivariate technique is used to distinguish the signal against the two major types of background. The two classifier shapes are combined to obtain a one dimensional shape where bin boundaries are defined to contain events having classifier scores within specified ranges.

\section{Single lepton $+b \bar{b}$ channel}

The $\mathrm{H} \rightarrow \mathrm{b} \bar{b}$ final state of the tHq process is divided in two categories depending on the b-jet multiplicity: 3-tag and 4-tag, where tag denotes the presence of b-tagged jets [12]. The selected events are also required to contain exactly one isolated lepton from semileptonic top decay and at least one un-tagged jet.

This channel is challenged with large $t \bar{t}+$ heavy-flavor background ( $\mathrm{t} \bar{t}+\mathrm{HF})$. A multivariate analysis is carried out to reconstruct kinematic properties of the event using jet-parton assignment, considering either tHq or $t \bar{t}$ hypothesis. These properties are used in a second multivariate analysis to discriminate the signal against the background. In addition, an opposite sign dilepton control region is selected for separating the $\mathrm{t} \bar{t}+\mathrm{HF}$ background from $t \overline{\mathrm{t}}+$ light-flavor $(\mathrm{t} \overline{\mathrm{t}}+\mathrm{LF})$ backgrounds and constrain them using simultaneous fit to data.

\section{Reinterpretation of the $H \rightarrow \gamma \gamma$ measurement}

The SM production of the $\mathrm{tH}$ process with $\mathrm{H} \rightarrow \gamma \gamma$ is also included in the Higgs boson analysis of the inclusive diphoton final state [13]. The tH events contribute in the " $\mathrm{t} \overline{\mathrm{H}} \mathrm{H}$ hadronic" and " $\mathrm{t} \mathrm{t} H$ leptonic" categories corresponding to fully hadronic or semileptonic decays of the top quarks in $\mathrm{t} \overline{\mathrm{t}} \mathrm{H}$ process.

The tH event yield and kinematic distributions are estimated using scale factors depending on the coupling modifier ratio $\kappa_{t} / \kappa_{V}$ which modifies the acceptance of signal events and the selection efficiency.

\section{Combination of different final states for extracting the tH signal}

The above analyses are combined using the statistical framework for Higgs combination [14]. The event selections of the individual channels are mutually exclusive, however, the systematic uncertainties due to common sources such as pileup, b-tag etc. have been considered as completely 
correlated. A combined maximum likelihood fit is performed on the one dimensional distribution from the multilepton channel, the event classifier discriminator distribution from the $b \bar{b}$ channel and the invariant diphoton mass distribution from the $\gamma \gamma$ final state analysis. The background-only hypothesis considered includes a $\kappa_{t}$ dependent $\mathrm{t} \overline{\mathrm{t}} \mathrm{H}$ contribution to extract the sensitivity to the $\mathrm{tH}$ process alone. The observed upper limit on the tH only cross section times branching fraction obtained for the Higgs boson decays to $\mathrm{WW}^{*} / \mathrm{ZZ}^{*} / \tau \tau / \mathrm{b} \bar{b} / \gamma \gamma$ in the SM (ITC) scenario is 25 (0.9) times the predicted value [16]. The observed and expected upper limits on the tH only signal strength is shown as a function of $\kappa_{t}$ for $\kappa_{V}=1.0$ on the left of Fig. 3.

The $95 \%$ CL uncertainties on a parameter of interest can be determined using the profile likelihood ratio test statistic [15], in which experimental and theoretical uncertainties are incorporated via corresponding nuisance parameters. In this analysis, constraints on $\kappa_{t}$ are derived from a distribution of the test statistic $-2 \Delta \ln \mathscr{L}$ or $-2\left[\ln \mathscr{L}\left(\kappa_{t}\right)-\ln \mathscr{L}\left(\hat{\kappa}_{t}\right)\right]$, where the $95 \%$ confidence interval covers values lying below 3.84 . Here the best-fit value is $\hat{\kappa}_{t}$ corresponding to the global maximum of the likelihood function. The corresponding $\kappa_{t}$ values are constrained inside the ranges (-0.9, $-0.5)$ and $(1.0,2.1)[16]$ as shown on the right of Fig. 3.
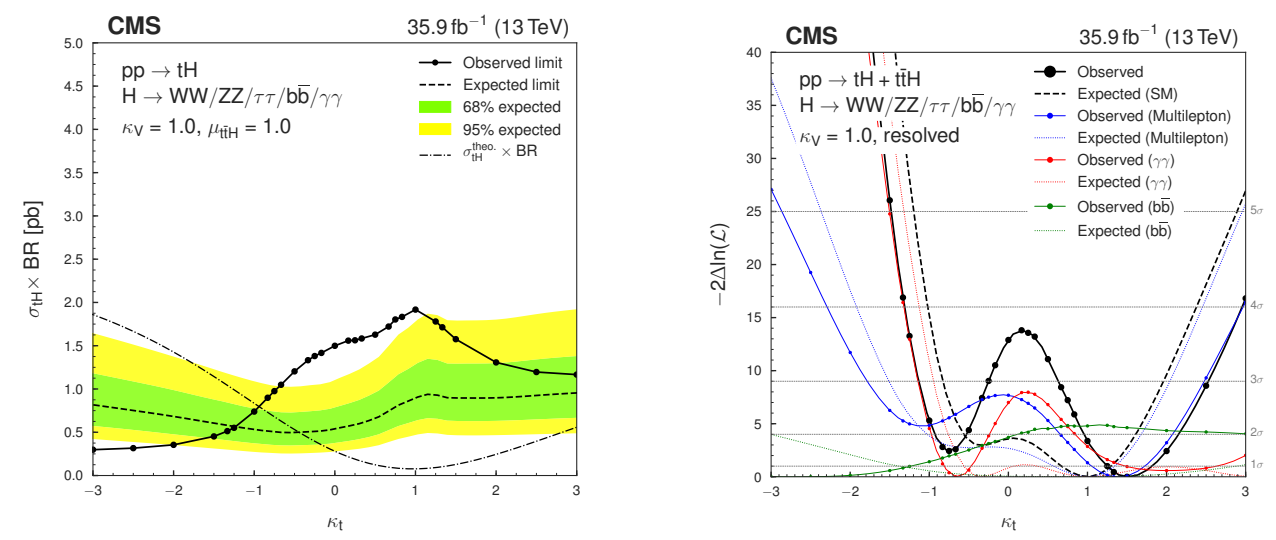

Figure 3: Observed and background-only expected cross section times branching ratio limits on combined tH production where $H \rightarrow W W^{*} / Z Z^{*} / \tau \tau / b \bar{b} / \gamma \gamma$, as a function of $\kappa_{t}$, for $\kappa_{V}=1.0$ [16] shown on the left. Scan of $-2 \Delta \ln \mathscr{L}$ vs $\kappa_{t}$ for the data (black line) and the individual channels (blue, red, and green), compared to the SM expectations (dashed lines) [16] shown on the right.

\section{Future prospects of the tH search}

From 2026 onwards, the LHC is expected to operate in the high luminosity mode, where the instantaneous luminosity is expected to reach up to $7.5 \times 10^{34} \mathrm{~cm}^{-2} \mathrm{~s}^{-1}$. A large amount of data $\left(3000 \mathrm{fb}^{-1}\right)$ will be collected during ten years of run time, paving experimental access to rare processes. However, high luminosity operation will also result in severe detector degradation in performance and high pileup rates, about $140-200$ per event, on average. Detector upgrades are therefore planned to mitigate the anticipated worsening of physics performance of the CMS experiment. The possible improvements in future results have been estimated during 2018 using simple projection scenarios with the help of available statistical methods, in the absence of reliable detector simulations [17]. 
In the simplest scenario, referred to as $\mathrm{S} 1$, it is assumed that all detector effects and systematic uncertainties remain the same as in the 2016 analysis; only the total integrated luminosity has been scaled. Theoretical uncertainties are reduced by a factor of $1 / 2$ and systematic uncertainties such as lepton identification, b-tagging efficiencies are scaled down as a function of integrated luminosity with a finite lower threshold, labelled as YR 2018 systematics or scenario S2. In both cases the statistical fluctuations of the simulation have been ignored. The analysis at high luminosity will gain from improving the theoretical uncertainties in estimating the background cross sections and also the non-prompt lepton contribution. An improvement by a factor of eight on the expected upper limit on the tH signal strength can be achieved with $3000 \mathrm{fb}^{-1}$ of data, as shown on the left of Fig. 4. Furthermore, it is expected that the future analyses will allow only positive values of $\kappa_{t}$, and with much more restricted range in the presence of a SM like signal. The corresponding negative log-likelihood scan of the test-statistics $q$ for the two scenarios at $3000 \mathrm{fb}^{-1}$ is shown on the right of Fig. 4.
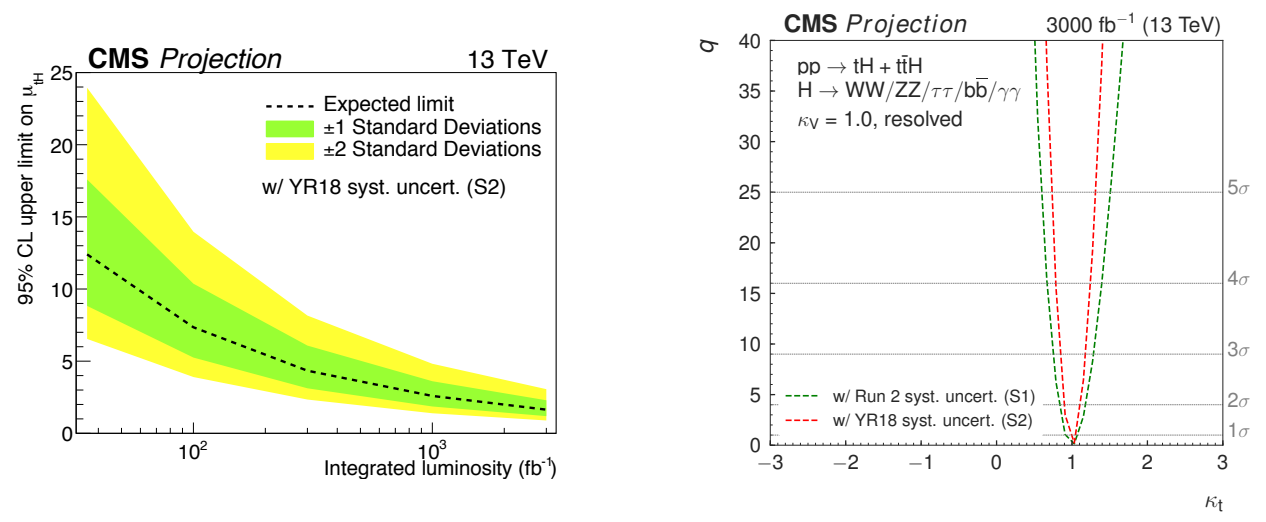

Figure 4: The variation of expected upper limit of the SM like point $\kappa_{t}=\kappa_{V}=1.0$ with integrated luminosity for projection scenarios [17] shown on the left. The right figure shows the variation of the test statistics for the two scenarios as a function of $\kappa_{t}[17]$.

\section{Conclusion}

The combined analysis using decay modes $H \rightarrow W W^{*} / Z Z^{*} / \tau \tau / b \bar{b} / \gamma \gamma$ based on 2016 data improves the earlier $8 \mathrm{TeV}$ results in terms of analysis techniques and sensitivity to the ITC production of the tHq process. Additional scenarios for anomalous Higgs boson couplings are explored to constrain the allowed ranges of $\kappa_{t}$ within $(-0.9,-0.5)$ and $(1.0,2.1)$ at $95 \%$ CL using a negative loglikelihood analysis. The sensitivity to the SM production of the $\mathrm{tH}$ process is still out of reach with the current dataset. A simple projection to HL-LHC luminosity indicates that the SM production will be accessible in the future.

It is to be noted that a dedicated diphoton channel analysis has yet to be performed which is the most sensitive to negative values of $\kappa_{t}$. In the era of precision physics, tH production plays a key role in the determination of the top-Higgs Yukawa coupling together with the t⿱一𫝀tH process. It remains to be verified how well the projection results compare with the actual LHC data: the future analyses may out-perform the prediction and achieve high precision as early as Run 3. 


\section{References}

[1] ATLAS Collaboration, G. Aad et al., "Observation of a new particle in the search for the Standard Model Higgs boson with the ATLAS detector at the LHC," Phys. Lett. B716 (2012) 1-29, arXiv:1207.7214 [hep-ex].

[2] CMS Collaboration, S. Chatrchyan et al., "Observation of a New Boson at a Mass of $125 \mathrm{GeV}$ with the CMS Experiment at the LHC,” Phys. Lett. B716 (2012) 30-61, arXiv:1207. 7235 [hep-ex].

[3] CMS Collaboration, S. Chatrchyan et al., "Observation of a New Boson with Mass Near $125 \mathrm{GeV}$ in pp Collisions at $\sqrt{s}=7$ and $8 \mathrm{TeV}$, JHEP 06 (2013) 081, arXiv:1303.4571 [hep-ex].

[4] ATLAS Collaboration, G. Aad et al., "The ATLAS Experiment at the CERN Large Hadron Collider," JINST 3 (2008) S08003.

[5] CMS Collaboration, S. Chatrchyan et al., "The CMS Experiment at the CERN LHC," JINST 3 (2008) S08004.

[6] ATLAS, CMS Collaborations, G. Aad et al., "Combined Measurement of the Higgs Boson Mass in pp Collisions at $\sqrt{s}=7$ and $8 \mathrm{TeV}$ with the ATLAS and CMS Experiments," Phys. Rev. Lett. 114 (2015) 191803, arXiv:1503.07589 [hep-ex].

[7] CMS Collaboration, V. Khachatryan et al., "Search for the associated production of a Higgs boson with a single top quark in proton-proton collisions at $\sqrt{s}=8 \mathrm{TeV}$," JHEP 06 (2016) 177 , arXiv:1509.08159 [hep-ex].

[8] P. Artoisenet et al., “A framework for Higgs characterisation,” JHEP 11 (2013) 043, arXiv:1306.6464 [hep-ph].

[9] F. Demartin, F. Maltoni, K. Mawatari, and M. Zaro, "Higgs production in association with a single top quark at the LHC," Eur. Phys. J. C75 no. 6, (2015) 267, arXiv:1504.00611 [hep-ph] .

[10] CMS Collaboration, A. M. Sirunyan et al., "Evidence for associated production of a Higgs boson with a top quark pair in final states with electrons, muons, and hadronically decaying $\tau$ leptons at $\sqrt{s}=13$ TeV," JHEP 08 (2018) 066, arXiv:1803.05485 [hep-ex] .

[11] CMS Collaboration, A. M. Sirunyan et al., "Search for production of a Higgs boson and a single top quark in multilepton final states in proton collisions at $\sqrt{s}=13 \mathrm{TeV}$," Tech. Rep. CMS-PAS-HIG-17-005, CERN, Geneva, 2017.

[12] CMS Collaboration, A. M. Sirunyan et al., "Search for the $\mathrm{tH}(\mathrm{H} \rightarrow \mathrm{b} \bar{b})$ process in pp collisions at $\sqrt{s}=13 \mathrm{TeV}$ and study of Higgs boson couplings," Tech. Rep. CMS-PAS-HIG-17-016, CERN, Geneva, 2018.

[13] CMS Collaboration, A. M. Sirunyan et al., "Measurements of Higgs boson properties in the diphoton decay channel in proton-proton collisions at $\sqrt{s}=13 \mathrm{TeV}$, , JHEP 11 (2018) 185, arXiv:1804.02716 [hep-ex].

[14] ATLAS, CMS, LHC Higgs Combination Group Collaboration, "Procedure for the LHC Higgs boson search combination in summer 2011”, CMS-NOTE-2011-005, ATL-PHYS-PUB-2011-011.

[15] G. Cowan, K. Cranmer, E. Gross, and O. Vitells, “Asymptotic formulae for likelihood-based tests of new physics,” Eur. Phys. J. C 71 (2011) 1554, arXiv:1007.1727 [physics.data-an] .

[16] CMS Collaboration, A. M. Sirunyan et al., "Search for associated production of a Higgs boson and a single top quark in proton-proton collisions at $\sqrt{s}=13 \mathrm{TeV}$," Phys. Rev. D99 no. 9, (2019) 092005, arXiv:1811.09696 [hep-ex]. 
[17] Physics of the HL-LHC Working Group Collaboration, M. Cepeda et al., "Higgs Physics at the HL-LHC and HE-LHC," arXiv:1902.00134 [hep-ph] .

[18] CMS Collaboration, A. M. Sirunyan et al., "Observation of t⿱亠幺十H production," Phys. Rev. Lett. 120 no. 23, (2018) 231801, arXiv:1804.02610 [hep-ex].

[19] ATLAS Collaboration, M. Aaboud et al., "Observation of Higgs boson production in association with a top quark pair at the LHC with the ATLAS detector," Phys. Lett. B784 (2018) 173-191, arXiv:1806.00425 [hep-ex]. 